8. Ne.

February 26, 1917. Reported sick on Balkan front : " marked haematuria." April 25

Examined at St. George's.

Vision of both eyes $6 / 6$. Both discs hyperaemic. Trace of fibrous tissue at upper temporal margins. Urine,

9. Sh. nothing to note. Convalescent.

\begin{abstract}
This patient showed a patch of atrophied choroid surrounded by pigment in periphery of left fundus, when examined six weeks after onset of nephritis. Subsequent examinations showed no change. In the absence of any recent signs the condition can hardly be attributed to the nephritis.
\end{abstract}

A survey of these cases shows :

First.-That neuro-retinal changes were not infrequent. The oedema is probably analogous in its pathology to renal oedema elsewhere. Had more cases been examined in the early stages, it would doubtless have been more frequently detected. Whether the grosser changes were merely a question of degree, or due to a different toxin, is quite obscure. Second.-That the prognosis as regards the restoration of the retinal function was excellent. Third.-That, granted the cases were more or less severe in type, and all of prolonged duration, the neuro-retinal changes were not of any additional serious prognostic import. This statement might readily require modification with a further knowledge of the ultimate course of the cases.

(To be continued.)

\title{
ON CASES OF ACUTE ANTERIOR ETHMOIDITIS IN YOUNG SUBJECTS
}

BY

Sydney Stephenson, LONDON.

IN my experience, the form of orbital suppuration described in this communication is tolerably common in young children, although there are grounds for believing that the essential nature of the condition is by no means always recognized. It is not rare for a young child or even a baby to be brought to the casualty department with a swelling of the lids of one eye, and a statement that he has been "out-of-sorts" for a day or two. Unless the two eyes are compared with respect to prominence, the fact is likely to be overlooked that the eye of the afiected side is more or less prominent. The condition goes unrecognized as an instance of orbital suppuration. Many of the milder cases get well under simple treatment, or, indeed, in the absence of any treatment. With or 
without a discharge of pus from the nostrils or into the nasopharynx, the orbital and associated symptoms subside forthwith. But a certain number of the cases find their way at a later stage into the eye department, with the obvious manifestations of what is rather loosely called " orbital cellulitis" or " orbital suppuration," as shown by tenderness, oedema, and redness of the eyelids, chemosis, and protrusion and impaired motility of the eyeball, which is usually displaced downward or downward and outward. The condition, in my experience, is always unilateral. General symptoms, as raised temperature, headache, vomiting, malaise, and so forth, are usually present in the more severe or acute cases. As a rule, there is no history of injury or of erysipelas, or, for that matter, of any illness, general or local, except such as may be reasonably supposed to arise from the orbital condition. I have been specially struck with the absence of any history of coryza in the patient.

I have become convinced from my clinical observations that the eye symptoms in the group of cases under discussion can be best explained by an acute inflammation of the anterior ethmoidal cells, although in certain of the more severe cases (case No. 9) it is impossible to exclude an inflammation of the posterior ethmoidal cells also. From their relative frequency, and the difficulty or impossibility of finding any adequate description of them, I am of the opinion that these cases deserve to be classed as a separate entity. Another reason is that when seen by the ophthalmic surgeon, usually somewhat.late in the attack, the diagnosis is almost certain to be that of " orbital cellulitis" or " orbital suppuration," without thought of what I am persuaded is the underlying factor.

So far as I have seen, the condition is not accompanied by any ophthalmoscopic signs, and in none of my cases has it been followed by atrophy of the optic disc. All the cases, however, were not examined in the early stages with the ophthalmoscope, and in some I had no opportunity of seeing the child after the acute symptoms had subsided. I have lost no patient from intra-cranial complications.

Rhinoscopic examination has been attempted in some instances, but the difficulties in children are considerable, a degree of expert knowledge (to which I can lay no claim) is advisable, and, all things considered, my results have amounted to very little.

Many of the cases get well under the simplest means. A cathartic (I am fond of prescribing a liberal dose, one drachm, of the compound jalap powder) followed by a continuous alcohol (6 per cent.) dressing over the lids of the affected eye bring many a case to a happy conclusion. Hot applications of boric acid often act well.

When symptoms are severe, or the general condition of the patient is threatening, surgical measures must be adopted. The usual procedure is to make an incision into the orbit over any spot which 
seems to indicate the presence of underlying pus. If nothing escapes, the dressing forceps, with closed blades, are introduced into the depths of the wound, and the blades of the instruments are then separated more or less widely. In some instances, even when Hilton's method is employed, the immediate result is negative or almost so (case No. 1), but pus makes its appearance two or three days after the operation. The explanation probably lies in the fact that early in the case there is no distinct abscess-cavity, while later the fluid lies beneath the periosteum and has not been liberated by the incision. Several experiences of this kind (which are disconcerting, to say the least) led me finally to adopt as a routine measure a somewhat different procedure. Under general anaesthesia, a curved incision down to the bone was made over the inner angle of the orbit, extending from the root of the nose to the inner end of the eyebrow, previously shaved, of course. A small raspatory was then introduced beneath the periosteum, and by its aid the latter was raised from the bone over the region of the anterior ethmoidal cells, when pus usually escaped. The method is essentially the same as the preliminary steps of Jansen's operation for the relief of suppuration in the frontal sinus. If the simpler orbital incision be preferred, I would urge that, in the absence of distinct indications to the contrary, the incision be made over the region of the anterior ethmoidal cells.

I quote details of the following ten cases, selected from among a larger number :

\section{Cases}

I.-Anterior ethmoiditis in a child of two years; recovery after operation; condition followed by unilateral amaurosis ; recovery of sight.

A bright boy, aged two years, developed, without known cause, upon January 20,1899, marked inflammatory oedema of the lids of the eye, so that they could not be opened voluntarily. The movements of the eyeball were abolished, and the globe was pushed forwards and outwards. The general symptoms were of a somewhat alarming character, for the child was semi-conscious, and evidently in great pain, the pulse was uncountable, and the temperature was $105^{\circ} \mathrm{F}$. On January 23, when the symptoms, both local and general, had become worse, a deep incision was made through the centre of the upper lid into the orbit, and a very small amount of pus escaped when dressing-forceps were introduced into the wound thus made. Three days later, and for some days after that, a considerable amount of pus was found upon the dressings used to cover the orbit. Inflammatory symptoms receded quickly. The most curious part of the case, however, has yet to be related. On January 30 , with a view to test the sight, the lids of 
the aftected eye were opened, the other eye meanwhile being covered up. As soon as this was done the child exclaimed, "Blind's down, daddy; all dark." The pupil was active, the media were clear, and the ophthalmoscopic appearances were normal. On another occasion, when asked where his nurse stood in the room, he said, "No; all dark." When the good eye was uncovered, however, he at once exclaimed, "Why, there's Norah!" (his nurse, who had been standing in front of him all the time). On February 17 it was noticed for the first time that the child could see with his right (affected) eye, the other being closed. This observation was confirmed next day by asking the child what he saw in his mother's hat. "Flowers and feathers" was the correct answer to this question. It was also found that the little boy could name various objects in his night-nursery without mistake or hesitation.

In 1906, when the lad was nine years of age, he was found to have V. of $6 / 5$ in each eye, with the total hypermetropia (ID.) corrected. The fundi were normal.

Although I knew that amaurosis might very occasionally follow prolonged blepharospasm, and have met with the condition after phlyctenulosis and interstitial keratitis, I was unaware until the foregoing case fell under my notice that the blindness might be limited to one eye.

2.-Anterior ethmoiditis in a child of four years, who had had a polypus removed from the nose. Resolution.

A male child, aged four years, was seen on October 20, 1908, with the history that twelve months previously a polypus had been removed from the nose at St. Thomas's Hospital, and that he had developed measles five months before I saw him. At 3 a.m. on October 19 the child awoke his parents, crying with pain in his head and right eye. Since then he had been ill.

Upon examination, the lids of the right eye were seen to be red and swollen, and the corresponding side of the face was slightly oedematous. The caruncle was reddened. The eyeball was proptosed, and deviated a little outwards. Some limitation of movement of the globe, especially upwards. The child was apathetic, and looked ill. Temperature $100.2^{\circ} \mathrm{F}$. It was noted that while the mucous membrane of the right nostril was reddened, that of the left nostril was pallid. No pus was found on rhinoscopic examination of the right middle meatus.

The orbital condition recovered after four days' rest in hospital, and treatment by purgatives and alcohol dressings.

3.-Acute anterior ethmoiditis in a child of four years following a contusion. Resolution.

A little boy, aged four years, was seen on March 1, 1903, at the Queen's Hospital for Children. The mother stated that the child 
bruised the left side of his face yesterday, but the eye seemed to be all right last night, On awakening this morning, the lids of the left eye were swollen, so that the child was brought to the hospital. On admission, the upper and the lower lid of the left eye were swollen, and the oedema extended slightly on to the neighbouring parts of the forehead and cheek. The skin was reddened, but not particularly tender. No signs of wound or bruising of the parts. On separating the swollen eyelids, definite proptosis was found, with limitation of movement in all directions. No tenderness upon pressure over the rim of the orbit. No marked redness of the ocular conjunctiva, and no secretion from the eye. Cornea clear; pupil active; no eflusion into the anterior chamber; ophthalmoscopic appearances normal. Child appears to be ill. Temperature $101^{\circ} \mathrm{F}$.

Calomel (grs. 2)was given. The swollen lids were smeared with mercurial ointment, and hot fomentations were applied to them for twenty minutes every four hours. On the following day, the temperature was $98^{\circ} \mathrm{F}$. and $98.4^{\circ} \mathrm{F}$. The swelling of the lids was reduced, and the parts were less painful. On March 4, three days after admission, there was no proptosis, and the eyelids could be readily opened, although the globe was displaced slightly downwards and outwards. There was a feeling of slightly increased resistance in the upper and inner part of the orbit. No fundus changes. On March 9 the movements and position of the eyeball were normal. The temperature had been practically normal since the 2nd instant. No thickening could be felt in the orbit. On March 13 lad was discharged from hospital, and when seen among the out-patients five days afterwards, nothing wrong could be found in the left orbit or eye.

4.-Acute ethmoiditis in a child of four and a half years. Resolution.

A lad, aged four and a half years, was first seen at the Evelina Hospital on December 27, 1907. There was a history of measles two months ago, of eczema of the scalp two weeks since, and of dischargefrom the left ear for one week. It appears that the childattended the casualty department of the hospital as the lids of the left eye were swollen, and the child was crying with pain in the affected eye. The following is an extract from the notes of the casualty officer: "Has been attending as a casualty for some days. There was at first a tremendous amount of oedema of the cellular tissue of the lids of the left side. Cellulitis. Several punctures made with a needle, and swelling diminished considerably under fomentations. Guttae atropinae were used."

The patient was first seen by me on December 31, 1907, when his condition was as under: Lids of left eye red, swollen, and closed, but not indurated. Eyeball slightly proptosed, and 
displaced somewhat downwards. Pulse 104. Temperature, $99.4^{\circ} \mathrm{F}$.

The child was admitted to the hospital, where a good deal of discharge from the left nostril was noted. Hot boric fomentations were applied every four hours to the lids of the aflected eye. On January 2, 1908, the urine was found to be acid, without deposit or albumin. On the following day there was great improvement in the state of the left eye. The fomentations were applied less frequently. On January 7-that is a week after admissionthere was no oedema of the eyelids, and so the fomentations were discontinued. On January 28 cure appeared to be complete. During the patient's sojourn in hospital it may be said that the temperature rose above $99^{\circ} \mathrm{F}$. twice only, namely, on January 1 , when at $10 \mathrm{p} . \mathrm{m}$. it reached $99.8^{\circ} \mathrm{F}$., and on January 12 , when the evening record stood at $101^{\circ} \mathrm{F}$. The pulse during the first five days after admission ranged from 80 to 130 a minute.

5.-Acute ethmoiditis in a child of six and a half months. Incision and evacuation of pus. Recovery.

This lad, aged six and a half months, was admitted to the Queen's Hospital for Children on October 19, 1907, on account of swelling of the lids of the right eye, first observed ten days previously. Breast fed. No history of injury. On admission the upper and lower lids of the right eye were found to be red, swollen, and tense. There were traces of mucopurulent secretion about the lashes, but beyond redness of the ocular conjunctiva no local condition could be found to account for it. There was definite proptosis and limitation in movement of the aftected eye. The cornea was clear. No disease of the naso-pharynx. No otorrhoea. Weight $16 \frac{1}{2} \mathrm{lbs}$. Temperature $99.8^{\circ} \mathrm{F}$. and $102.2^{\circ} \mathrm{F}$.

One grain of calomel was given, and hot lead lotion was constantly applied to the lids of the afiected eye. On October 30 (eleven days after admission) the swelling of the lids was reduced. There was well-marked proptosis of the right eye. A fold of oedematous conjunctiva could be seen on everting the upper lid of the right eye, There was some slight redness of the outer canthus of the affected eye. On November 1 (12 days after admission to hospital) a curved incision, about one inch in length, was made parallel to and immediately beneath the inner end of the upper margin of the orbit under chloroform. Sinus forceps were introduced, and when their blades were separated, about a teaspoonful of pus was evacuated. The orbital roof felt bare. A drainage tube was inserted into the wound. On November 14 there was still slight proptosis, but the eyelids could be opened at will. There was a depressed cicatrix beneath the inner third of the arch of the right orbit, along with a sinus at the bottom of the cicatrix. Child able to open the eye, although the upper lid was still rather swollen. No 
proptosis, but the eyeball was displaced downwards. General condition good. It may be noted that the temperature $\left(99.8^{\circ} \mathrm{F}\right.$. at the time of operation) has exceeded the normal once only since the operation, namely, on the evening of November 10 , when it reached $99^{\circ} \mathrm{F}$. On November 20 the child opened the lids of the right eye nearly as well as those of the left. There was still some oedema of the right upper lid. No proptosis, and little displacement of the globe downwards. Fundi (examined under homatropin) normal. No obvious disease in the naso-pharynx or nose. On November 27, after a stay of thirty-nine days in hospital, patient discharged. The surgical cicatrix was then firm, and showed no signs of discharge.

On February 6, 19018, being then ten months of age, patient was seen among the out-patients. There was a linear cicatrix, $20 \mathrm{~mm}$. long, starting from the inner end of the orbit and extending over the eye. Its inner end was slightly puckered, and beneath the pucker a small hard lump was to be felt in the orbit over the position of the ethmoidal labyrinth. Slight ptosis of the right upper lid, and fulness of "covering fold." The right eye, although not proptosed and possessing good movements, was slightly divergent. The sight appeared to be good.

6.-Acute ethmoiditis in a girl three and a half years. Resolution.

A female, aged three and a half years, was seen on January 7 , 1908 , with the statement that she had been "poorly " for a fortnight, and that her left eye became affected on the 5th instant. The pulse was 120 , and the temperature $100^{\circ} \mathrm{F}$. There was mild follicular tonsillitis, and the glands on both sides of the neck were enlarged. The lids of the left eye were ecchymotic, but not swollen. Definite but not marked proptosis. Movements of globe deficient in all directions. No chemosis. Anterior segment of eyeball looked normal.

Child admitted to hospital, and one drachm of compound jalap powder given. The lids of the affected eye were treated with hot lead lotion, kept continuously applied. After a stay in hospital of ten days, the child was discharged. The movements of the left eye were then normal, and not a trace remained of the former ecchymosis.

7.-Acute ethmoiditis in a boy of seven years. Discharge of pus from the nose. Resolution.

A boy, aged seven years, was seen among the out-patients at the Kensington General Hospital in March, 1907, with symptoms of acute anterior ethmoiditis, of about thirty hours' duration. The temperature was $99^{\circ} \mathrm{F}$., the pulse was 130 , and the lad seemed ill. He was referred to the Queen's Hospital for Children, where he remained as an in-patient from March 6, 1907, until March 27, 1907, when he was discharged well. 
On admission, the upper and lower lids of the right eye were swollen, and on separating them, the globe was seen to be pushed forwards and somewhat downwards. Slight conjunctival redness. There was photophobia, and some muco-purulent discharge among the eyelashes. The movements of the globe were very limited in all directions. The anterior segment of the eye showed no obvious changes, and the ophthalmoscopic appearances were normal. The pupil was of medium size, and reacted well to light and to convergence. The temperature was $100.8^{\circ} \mathrm{F}$. Weight, $39 \frac{1}{2} \mathrm{lbs}$. There were several carious teeth in both jaws.

A purgative (Pulv. jalapae co.) was given. On the day following admission the temperature, $100.4^{\circ} \mathrm{F}$. in the morning, had fallen to $97.8^{\circ} \mathrm{F}$. at night, and this coincided with quite a quantity of pus, which had presumably escaped from the nose, being found on the child's pillow. A week later (March 14, 1907) the lids of the right eye were rather oedematous and semi-closed. Slight proptosis. No definite changes in the nares. Pupil reacted to light. Vision appeared to be good. Temperature has once only exceeded $99^{\circ} \mathrm{F}$. since last note, namely, on the 12 th, when the evening record was $99.2^{\circ} \mathrm{F}$. On March 19 th several carious teeth were removed. On March 27, after a stay in hospital of three weeks, patient discharged as cured.

8.-Acute ethmoiditis in a boy of twelve years. Resolution.

A boy of twelve years was first seen at the Queen's Hospital for Children on July 23, 1908, with the history that he awoke four days ago with pain in the right eye and corresponding side of the brow. The lids became red, and yesterday, when the pain got very great, it was noticed that the eye was pushed forward. On admission, it was observed that the lids of the right eye were in a state of inflammatory oedema, and that the eye was proptosed. Considerable limitation of movement of the eyeball. No redness of the conjunctiva. Pupil active, and V. 5/6.

The case was treated with phenacetin to relieve pain, boric fomentations to the swollen eyelids, and one drachm of the syrup of the iodide of iron was administered three times a day. When seen a week later, the lad was practically well. There was no proptosis, and the movements of the eyeball were good. The boy, however, had complained of diplopia. When seen again on September 17, 1908, there were complaints of difficulty in sight, and of some pain in the right eye. The correction of slight hypermetropic astigmatism by glasses seemed to render him quite comfortable.

9.-Severe acute ethmoiditis in a baby of fourteen days. Incision. Recovery.

R. M., born on January 18, 1909, developed swelling of the lids of the left eye on February 1, 1909, and next day the 
corresponding eye seemed pushed out of position. There had been no discharge from the eye since the baby was born. Patient is the eighth in the family. The first four children died within four or five days of birth. The fifth child died at two and a half years from meningitis. The sixth and seventh child, aged respectively three and two years, are alive and healthy.

On admission, the lids of the left eye are red and oedematous, and the globe protrudes, so as to lie between the eyelids. The movements of the eyeball, although abolished laterally, are still present slightly in an upward and downward direction. The ocular conjunctiva is oedematous and overlaps the cornea slightly, but is of pale colour. No mastoid oedema. Pupil active to light. There is no discharge from the nose. The fundus oculi appears to be normal. Temperature, $101^{\circ} \mathrm{F}$.

The baby was taken into the hospital, where castor oil was given, and boric fomentations were applied to the swollen eyelids. On February 4, 1909, the following note was made.- "Left eye. Inflammatory oedema upper and lower lid, the skin being of livid colour. Marked proptosis, practically in the axis of the orbit. Cornea covered by eyelids. Non-inflammatory swelling of the ocular conjunctiva. General condition of the baby fair. Temperature, $102^{\circ} \mathrm{F}$. to $100^{\circ} \mathrm{F}$. Bowels opened five times. Navel in good condition : no obvious source of sepsis. Nurse noticed pus running from the left nostril this morning."

February 5, 1909.-The proptosis has increased, and lids now scarcely cover the cornea. Slight purulent discharge from the left nostril. Under chloroform, an incision, about half an inch long, was made along the inner third of the upper margin of the orbit. A blunt director was introduced to the back of the orbit along the nasal side of the orbital cavity, but no pus was evacuated. The base of the inner wall seemed rough and bare, and readily yielded to the pressure of the probe. At this stage some discharge occurred from the left nostril, but soon ceased. A second incision was then made at the lower-inner margin of the orbit, and the director was again introduced. A quantity of yellowish-green pus, estimated at one or two teaspoonfuls, was evacuated. The inner surface of the floor seemed bare and rough. The upper wound was left open, and the lower wound plugged. Fomentations were applied. Temperature prior to operation, $100^{\circ} \mathrm{F}$. and $100^{\circ} \mathrm{F}$., was $99^{\circ} 2^{\circ} \mathrm{F}$., $100^{\circ} 6^{\circ} \mathrm{F}$., and $984^{\circ} \mathrm{F}$.

February 6, 1909.-There is a copious discharge of greenishbrown pus from the lower incision in the orbit. Bowels freely open. Temperature, $102^{\circ} \mathrm{F}$., $100^{\circ} 6^{\circ} \mathrm{F}$., and $99^{\circ} 4^{\circ} \mathrm{F}$. February 7, 1909.-Oedema of lids and proptosis less marked. The movements of the eyeball are now tolerably free. Temperature, $100 \cdot 2^{\circ}$ $\mathrm{F}$ and $98^{\circ} 4^{\circ} \mathrm{F}$. February 8.- Proptosis and oedema considerably 
reduced, and the movements of the eyeball in all directions increased in amplitude. The fold of chemotic conjunctiva which protrudes from between the lids, shows some tendency to superficial ulceration. There is a small, grey opacity just below the centre of the cornea. Temperature $99^{\circ} \mathrm{F}$. and $98^{\circ} \mathrm{F}$. On February 9.A note was made to the eftect that the condition was much better. Temperature $99^{\circ} \mathrm{F}$., and $98^{\circ} \mathrm{F}$. A fine probe still traverses the floor of the orbit to the inner wall, which is distinctly carious. February 11, 1909.-Proptosis now little marked, and the movements of the eyeballs are almost complete in all directions except outwards. There is a thick discharge from the lower wound and the left nostril, and pus can be seen in the middle meatus of the left side. The ocular conjunctiva is almost normal, although there remains a thickened fold on the left side of the cornea. The conjunctival culs-de-sac are slighly injected. Weight $61 \mathrm{lb}$. Temperature $99.8^{\circ} \mathrm{F}$. and $98^{\circ} \mathrm{F}$. On February 17 it was noted that discharge from the lower orbital wound had ceased, although purulent discharge from the left nostril still persisted. Slight proptosis and oedema of the eyelids present. On February 26 a smooth, round swelling which had developed at the posterior border of the anterior fontanelle was opened and found to contain pus. On March 12, after a stay of thirty-eight days, the child was made an out-patient. On May 6, 1909, the baby, now aged $3 \frac{1}{2}$ months, seemed to be well. No proptosis or squint, and movements of eye free.

10.-Acute ethmoiditis in a boy of three and a quarter years. Two operations. Recovery.

On June 25, 1902, I was asked by Dr. Macdonald Brown to see a boy, aged three and a quarter years. The following history was given to me: The child awoke yesterday morning complaining of headache and showing some redness and swelling of the lids of the left eye. That evening the pulse was 160 and the temperature $102^{\circ} \mathrm{F}$. No cerebral symptoms.

On examination, there was marked inflammatory oedema of the lids of the left eye. Slight proptosis. Globe displaced downwards. The movements of the eyeball were defective. A good fundus reflex. With the affected eye the child could recognise an object held near him. The side of the face was a little swollen. No tonsillar or dental mischief. No obvious source of sepsis. No history of any zymotic ailment.

When seen on the next day (June 26) the symptoms were more pronounced. Temperature, $101^{\circ} \mathrm{F}$. to $103^{\circ} \mathrm{F}$. No mastoid oedema. More proptosis and redness and oedema of the left eyelids. Chemosis opposite the palpebral fissure. Fluctuation was thought to be present in the orbit above the left eye. Under chloroform, a curvilinear incision was made under the centre of the supra-orbital 
ridge, and dressing-forceps were introduced into the wound and a little pus, mixed with blood and serum, escaped. A small drainage tube was introduced into the centre of the wound, the rest of which was sutured. On the following day, June 27, the oedema of the lower lid and corresponding side of the face was more marked. Some pus was stated to have escaped by the drainage tube, the mouth of which also contained similar fluid. Mr. Percy $\mathrm{H}$. Dean, who was called in consultation, deepened the original incision and found pus, not in great quantity, at the upper and inner part of the orbit. He next incised the lewer lid, at the inner end of which he also found and evacuated pus. The bone in the last-named position was bare. A drainage tube, was inserted through the lower wound, and a piece of gauze was left in the upper wound. Finally, the wounds were closed with sutures.

I did not have the opportunity of seeing the patient again, but I learned from Dr. Macdonald Brown that recovery was complete.

\title{
Conclusions
}

1. In young subjects, usually under five years of age, a form of orbital inflammation or suppuration is not infrequent.

2. The condition is always unilateral.

3. There are clinical grounds for believing that an acute inflammation of the anterior ethmoidal cells is the primary condition.

4. The prognosis is good, since the condition undergoes resolution often without surgical intervention.

\section{A CASE OF HYALINE BODIES ON THE OPTIC DISC}

\author{
BY \\ G. VineR, Capt. R.A.M.C.
}

(With plate.)

No. 34844 Gnr. W-, Australian Field Artillery, aged 38, admitted to Ophthalmic Department, No. 3 General Hospital, France, November 14, 1917, with a diagnosis of shell wound of left eye.

On examination, there was a contused wound with much ecchymosis, situated on the left upper lid and eyebrow, close to the outer canthus; no foreign body present in wound and no fracture detected. Left globe intact, and all external appearances normal. L.V. $=6 / 24$ c. +1.00 D. cyl. axis vert. $=6 / 12$. Field of vision full except in lower and nasal quadrant, where it is contracted to $45^{\circ}$. No central scotoma, but the blind spot appears to be distinctly larger than normal. 